\title{
The effect of tides on near-core rotation: analysis of 35 Kepler $\gamma$ Doradus stars in eclipsing and spectroscopic binaries
}

\author{
Gang Li, ${ }^{1,2 \star}$ Zhao Guo, $^{3,4 \star}{ }^{\text {Jim Fuller }}{ }^{\circledR}, 5$ Timothy R. Bedding ${ }^{\circledR},{ }^{1,2 \star}$ Simon J. Murphy ${ }^{\circledR},{ }^{1,2}$ \\ Isabel L. Colman ${ }^{1,2}$ and Daniel R. Hey ${ }^{1,2}$ \\ ${ }^{1}$ Sydney Institute for Astronomy (SIfA), School of Physics, University of Sydney, NSW 2006, Australia \\ ${ }^{2}$ Stellar Astrophysics Centre, Department of Physics and Astronomy, Aarhus University, Ny Munkegade 120, DK-8000 Aarhus C, Denmark \\ ${ }^{3}$ Department of Astronomy and Astrophysics, 525 Davey Laboratory, The Pennsylvania State University, University Park, PA 16802, USA \\ ${ }^{4}$ Center for Exoplanets and Habitable Worlds, 525 Davey Laboratory, The Pennsylvania State University, University Park, PA 16802, USA \\ ${ }^{5}$ TAPIR, Mailcode 350-17, California Institute of Technology, Pasadena, CA 91125, USA
}

Accepted 2020 July 26. Received 2020 July 24; in original form 2020 May 30

\begin{abstract}
We systematically searched for gravity- and Rossby-mode period spacing patterns in Kepler eclipsing binaries with $\gamma$ Doradus pulsators. These stars provide an excellent opportunity to test the theory of tidal synchronization and angular momentum transport in F- and A-type stars. We discovered 35 systems that show clear patterns, including the spectroscopic binary KIC 10080943. Combined with 45 non-eclipsing binaries with $\gamma$ Dor components that have been found using pulsation timing, we measured their near-core rotation rates and asymptotic period spacings. We find that many stars are tidally locked if the orbital periods are shorter than $10 \mathrm{~d}$, in which the near-core rotation periods given by the traditional approximation of rotation are consistent with the orbital period. Compared to the single stars, $\gamma$ Dor stars in binaries tend to have slower near-core rotation rates, likely a consequence of tidal spin-down. We also find three stars that have extremely slow near-core rotation rates. To explain these, we hypothesize that unstable tidally excited oscillations can transfer angular momentum from the star to the orbit, and slow the star below synchronism, a process we refer to as 'inverse tides'.
\end{abstract}

Key words: binaries: eclipsing-stars: interiors -stars: oscillations-stars: rotation.

\section{INTRODUCTION}

Binary and multiple systems are common, probably outnumbering single stars throughout much of the Hertzsprung-Russell (HR) diagram (Duchêne \& Kraus 2013; Moe \& Di Stefano 2017; Guszejnov, Hopkins \& Krumholz 2017; Murphy 2018). Eclipsing binaries provide important tests for calibrating stellar parameters, giving stellar masses and radii to an accuracy of $\sim 2$ per cent (Andersen 1991) and, in ideal cases, to 0.2 per cent (Maxted et al. 2020). This also improves the accuracy of the parameters of orbiting planets (e.g. Doyle et al. 2011). Well-measured stellar parameters in binary systems allow us to test stellar evolution models or study mass transfer (e.g. Faulkner 1971; Torres \& Ribas 2002; del Burgo \& Allende Prieto 2018), and determine distances (e.g. Hilditch, Howarth \& Harries 2005; North et al. 2010).

A critical question about binarity is how tides affect the system. In a binary system with a radiative-envelope main-sequence star, the periodic gravitational disturbance by its companion star can excite oscillations and lead to circularization and synchronization (Zahn 1975, 1977). Radiative damping of low-frequency tidal oscillations can lead to angular momentum exchange between orbital motion and stellar rotation, or orbital energy dissipation into thermal energy, or

\footnotetext{
^E-mail: gali8292@uni.sydney.edu.au (GL); fffeynman@gmail.com (ZG);
} tim.bedding@sydney.edu.au (TRB) angular momentum transfer by the tidally excited oscillations (e.g. Goldreich \& Nicholson 1989a, b; Lee 1993).

In this work, we report observations of the near-core rotation rates of 35 binaries, which are important for understanding tidal effects in binary systems. We use self-excited gravity modes to measure the near-core rotation rates in $\gamma$ Doradus stars, which are mainsequence stars with masses between 1.4 and 2.0 solar masses (Balona, Krisciunas \& Cousins 1994; Kaye et al. 1999). The gravity modes carry information about the near-core regions, such as the chemical composition gradient (Miglio et al. 2008), and the near-core rotation rate (Bouabid et al. 2013; Ouazzani et al. 2017), hence allow us to infer conditions in the stellar interiors. Thanks to the high-precision continuous photometry from the Kepler space mission (Borucki et al. 2010; Koch et al. 2010), we can resolve the gravity modes in over $600 \gamma$ Dor stars (Li et al. 2019b, 2020). Most show dipole sectoral $\mathrm{g}$ modes with decreasing period spacings, which are the period differences between two consecutive modes (e.g. Van Reeth et al. 2015; Li et al. 2019b). The typical near-core rotation rate is around $1 \mathrm{~d}^{-1}$ (Li et al. 2020), measured by the traditional approximation of rotation (TAR, e.g. Eckart 1960; Lee \& Saio 1997; Townsend 2003; Van Reeth, Tkachenko \& Aerts 2016; Li et al. 2020). Somewhat surprisingly, there are also many slowly rotating $\gamma$ Dor stars, showing nearly identical period spacings or rotational splittings (e.g. Kurtz et al. 2014; Saio et al. 2015; Keen et al. 2015; Murphy et al. 2016a; Li et al. 2019a, 2020). 
In addition to g modes, we also see Rossby modes ( $\mathrm{r}$ modes) in about one-fifth of the $\gamma$ Dor stars (Van Reeth et al. 2016; Li et al. 2019b, 2020). These modes are restored primarily by the Coriolis force (e.g. Rossby 1939; Papaloizou \& Pringle 1978; Lee \& Saio 1997; Saio et al. 2018a), have pulsation periods longer than the rotation period in the observer's frame, and show increasing period spacing patterns as a function of period (Provost, Berthomieu \& Rocca 1981; Saio 1982). We can use g and $\mathrm{r}$ modes together to measure the near-core rotation rates. When a star rotates rigidly, both $\mathrm{g}$ and $\mathrm{r}$ modes are sensitive to the inner regions. However, if the core rotates more rapidly than the surface, which is consistent with the theory and observation (e.g. Rieutord 2006; Hypolite \& Rieutord 2014; Li et al. 2020), r modes will be more sensitive to the outer layers, whereas the g modes are more sensitive to the nearcore regions. This situation is rare in the observations but provides a new approach to detect radial differential rotation (Van Reeth et al. 2018). Rossby modes with small period spacings are still unresolved by the 4-yr Kepler data, hence they show an amplitude hump near the rotation frequency (e.g. Saio et al. 2018a). These humps offer a new opportunity to measure the rotation rates of stars, including stars in eclipsing binaries (Saio 2019).

In this work, we report our study of $\gamma$ Dor stars in eclipsing binaries that show clear period spacing patterns. In Section 2, we describe the data reduction method, including the eclipse removal, detection of period spacing patterns, and the TAR fitting. We show the results and discussions in Section 3. Finally, we make conclusions in Section 4.

\section{DATA REDUCTION}

\subsection{Eclipse removal}

We downloaded 4-yr Kepler long-cadence (29.45-min sampling) simple aperture photometry light curves using the PYTHON package LIGHTKURVE (Lightkurve Collaboration et al. 2018). We applied a high-pass filter to the light curves to remove any slow trend, during which the flux was divided by the median in a 10-d moving window.

We measured the times of primary eclipses and calculated the differences from a linear ephemeris to determine the best orbital periods (e.g. Sterken 2005). The procedure involved the following steps: given an initial time of the first primary light minimum $T_{0 \text {, ini }}$ and initial orbital period $P_{\text {orb, ini }}$, we searched for the eclipses around the calculated times of light minima $C=T_{0 \text {, ini }}+E P_{\text {orb, ini, where } E}$ is the integer cycle number. We fitted a parabola to the local light curves to get the observed times of light minima (' $O$ '). The slope of the ' $O$ minus $C$ ' versus cycle number was used to corrected the initial orbital period. The light curves were folded by the orbital periods to obtain the phased light curves, and the latter were rebinned to generate the binned light curves. The bin sizes were small enough to assure that the eclipses were well sampled. The binned light curves were subtracted from the original light curves to remove the eclipses, and the residuals were used for forward seismic analysis.

Fig. 1 shows the result of the eclipses removal, using KIC 3228863 as an example. The binary system and its third component were studied using the Kepler photometry and ground-based spectroscopy by Lee et al. (2019). Our calculated period is $0.73094425 \pm$ $0.00000004 \mathrm{~d}$, consistent with their measurement. In the top panel, we display seven days of the Kepler data after the normalization and detrending, where the primary eclipses block about 40 per cent of the total flux. We also plot the binned light curve. The residuals between the raw data and the binned light curve are shown in the bottom panel, where the $\gamma$ Dor-type pulsations are revealed with a total amplitude around 1 per cent.
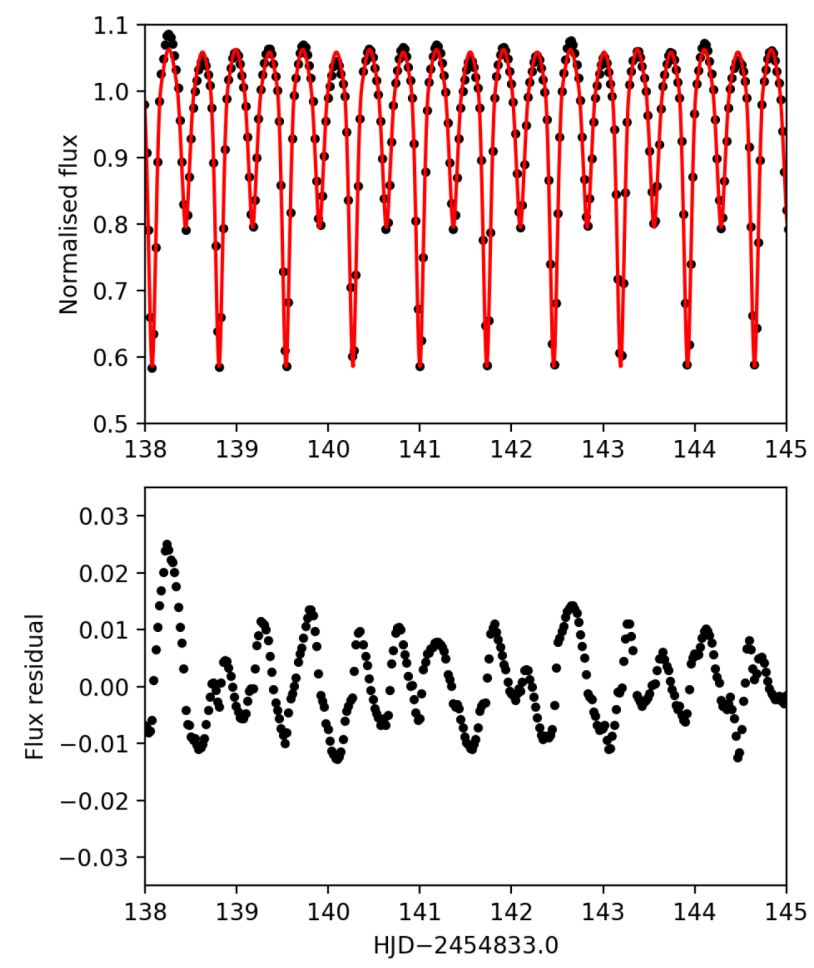

Figure 1. The light curve of KIC 3228863. Top: the light curve before removing the eclipses. The $x$-axis is the Kepler Barycentric Julian Day (BKJD), which is a Julian day minus 2454833.0. The $y$-axis is the normalized flux. The black dots are the raw flux data, while the red line is the binned light curve. Bottom: the light curve residual.

Some eclipsing binaries have eccentric orbits. For those whose phases of secondary light minima are around 0.5 , we assumed that their eccentricities are equal to zero. This assumption is reasonable for short-period binaries since they typically show circular orbits (Shporer et al. 2016). For the long-period eccentric binaries, the light minima at phase near 0.5 only appear when the longitude of periastron is near $90^{\circ}$, which is not likely to happen (see equation 18 in Matson et al. 2016). For those with obvious eccentricity, we checked the previous literature and collected their eccentricities.

In an eccentric orbit, true synchronous rotation is impossible, so a 'pseudo-synchronous' period $P_{\mathrm{ps}}$ is calculated instead. For this, we used equation (42) in Hut (1981):

$P_{\mathrm{ps}}=P_{\mathrm{orb}} \frac{\left(1+3 e^{2}+\frac{3}{8} e^{4}\right)\left(1-e^{2}\right)^{3 / 2}}{1+\frac{15}{2} e^{2}+\frac{45}{8} e^{4}+\frac{5}{16} e^{6}}$,

where $e$ is the eccentricity and $P_{\text {orb }}$ is the orbital period. Pseudosynchronous spin can lead to a zero net torque during each orbit, hence the spin will not evolve (e.g. Hut 1981; Welsh et al. 2011).

\subsection{Oscillation signal extraction}

We calculated the amplitude spectra of the residual light curves where the eclipses have been removed. The frequencies were obtained by the pre-whitening algorithm described by Li et al. (2019a), with the criterion that the signal-to-noise ratio $(\mathrm{S} / \mathrm{N})$ should be larger than 3.5. Although the well-accepted criterion is $\mathrm{S} / \mathrm{N}>4$ (Breger et al. 1993), we still adopted the peaks which have slightly smaller S/N but follow the patterns. 

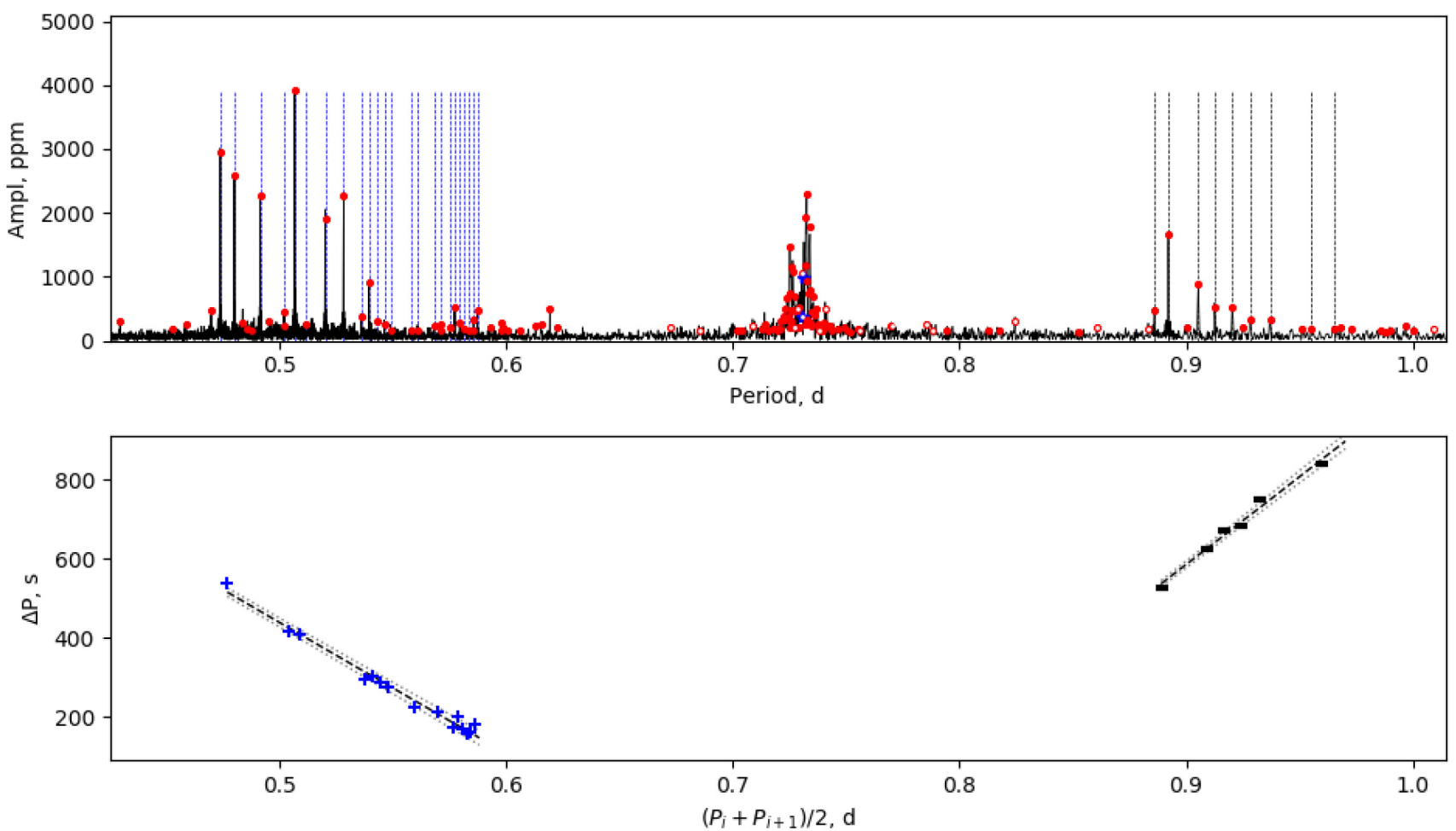

Figure 2. Top: the amplitude spectrum of KIC 3228863 after removing the eclipses. The blue vertical dashed lines on the left show the peaks of $l=1, m=$ $1 \mathrm{~g}$ modes. The black vertical dashed lines on the right mark the peaks of $k=-2, m=-1 \mathrm{r}$ modes. The hump at $\sim 0.73 \mathrm{~d}$ might be the surface modulation signal. Bottom: the period spacings of $\mathrm{g}$ and $\mathrm{r}$ modes. The blue crosses are the pattern of $l=1, m=1 \mathrm{~g}$ modes and the black symbols are those of $k=-2, m=$ $-1 \mathrm{r}$ modes. The dashed lines show the best-fitting linear relation, whose uncertainties are shown by the grey dotted lines surrounding them.

The g- and r-mode patterns were identified by the algorithm described by Li et al. (2019a). Mode identification was made based on the slope-period diagram founded on the $611 \gamma$ Dor stars by $\mathrm{Li}$ et al. (2020), where the patterns clustered into different groups due to different kinds of modes. Fig. 2 displays the amplitude spectrum and oscillation patterns of KIC 3228863. Having removed the eclipses, we find clear oscillation patterns of $\mathrm{g}$ and $\mathrm{r}$ modes. We also find a hump at the orbital period $\sim 0.73 \mathrm{~d}$, which could be caused by variations of the eclipses that prevented them from being totally removed. Mechanisms that can cause such eclipse variations include: apsidal motion (e.g. Hambleton et al. 2013), spot evolution and motion (e.g. Sriram et al. 2017; Czesla et al. 2019), or instrumental effects.

We fitted the period spacings in each star using the TAR to obtain the near-core rotation rates (Van Reeth et al. 2016; Li et al. $2019 \mathrm{~b}, 2020)$. The pulsation periods in the corotating frame are given by

$P_{n l m, \mathrm{co}}^{\mathrm{TAR}}=\frac{\Pi_{0}}{\sqrt{\lambda_{l, m, s}}}\left(n+\varepsilon_{g}\right)$,

where $\Pi_{0}=2 \pi^{2}\left(\int \frac{N}{r} \mathrm{~d} r\right)^{-1}$ is the asymptotic period spacing, $N$ is the buoyancy frequency, $n$ is the radial order, and the phase term $\varepsilon_{g}$ was fixed as 0.5 . The phase term does not change the result since the radial orders are typically high $(\sim 50)$, and we fit the period spacings rather than individual periods. The symbol $\lambda_{l, m, s}$ is the eigenvalue of the Laplace tidal equation, which is specified by the angular degree $l$ for $g$ modes or the value $k$ for $r$ modes, the azimuthal order $m$, and the spin parameter $s$ (Eckart 1960; Lee \& Saio 1997; Townsend 2003; Saio et al. 2018a, b). The value $k$ is equal to $l-|m|$ in g modes. For $\mathrm{r}$ modes, $k$ is used because the angular degree $l$ is undefined
(Lee \& Saio 1997), and an even (odd) $|k|$ corresponds to temperature perturbations symmetric (antisymmetric) to the equator. We used the convention that positive values of $m$ denote prograde modes. Most $\gamma$ Dor stars rotate rapidly, and in this situation for prograde sectoral $(l=m) \mathrm{g}$ modes we have

$\lambda_{l=m} \approx m^{2}$,

and for $k \leq 2 \mathrm{r}$ modes,

$\lambda_{k \leq 2} \approx m^{2}(2|k|-1)^{-2}$,

which are the most commonly observed modes (Berthomieu et al. 1978; Townsend 2003; Saio et al. 2018b; Takata et al. 2020). Accurate values of $\lambda$ can be obtained from the stellar oscillation code GYRE (Townsend \& Teitler 2013; Townsend, Goldstein \& Zweibel 2018). The spin parameter is defined as

$s \equiv \frac{2 f_{\text {rot }}}{f_{\text {co }}}$,

where $f_{\text {rot }}$ is the rotation frequency and $f_{\text {co }}$ is the pulsation frequency in the corotating frame. After calculating the pulsation periods in the corotating frame by equation (2), the TAR frequency in the inertial reference frame is given by

$f_{n l m, \text { in }}^{\mathrm{TAR}}=1 / P_{n l m, \mathrm{co}}^{\mathrm{TAR}}+m f_{\mathrm{rot}}$,

and the period in the observer's reference frame is the inverse of the frequency. Finally, we can obtain the near-core rotation rates, the asymptotic spacings, and the radial orders by comparing the observed and calculated period spacing patterns.

For example, we applied our TAR fitting algorithm to KIC 3228863 and found that its near-core rotation rate is $1.3716 \pm$ 
$0.0013 \mathrm{~d}^{-1}$. The ratio between the near-core rotation period and the orbital period is $0.9975 \pm 0.0009$, showing that the near-core region of KIC 3228863 rotates synchronously with the orbital motion.

\section{RESULTS}

\subsection{Sample selection}

Gaulme \& Guzik (2019) reported $115 \gamma$ Dor stars by conducting a systematic search for stellar pulsators in all the Kepler eclipsing binaries from the Villanova EB data base ${ }^{1}$ (e.g. Prša et al. 2011; Slawson et al. 2011; Matijevič et al. 2012; Conroy et al. 2014; Kirk et al. 2016). We searched for g- and r-mode patterns in these $115 \gamma$ Dor stars and also in the $\gamma$ Dor binaries reported in previous literature.

The known $\gamma$ Dor binaries whose period spacings were not previously measured are: KIC 3228863 (Lee et al. 2019, 2014); KIC 4150611 (Hełminiak et al. 2017); KIC 6048106 (Lee 2016; Samadi Ghadim, Lampens \& Jassur 2018); KIC 6206751 (Lee \& Park 2018); KIC 8569819 (Kurtz et al. 2015); KIC 9236858 (Kjurkchieva \& Atanasova 2016); and KIC 9851944 (Guo et al. 2016). These works focused mainly on the dynamics of the binary systems, hence they did not measure the period spacings of g modes.

The known $\gamma$ Dor binaries whose period spacings have already been measured are: KIC 3341457 (Li et al. 2019b, 2020); KIC 4142768 (Guo et al. 2019); KIC 7385478 (Guo \& Li 2019; Özdarcan \& Dal 2017); KIC 8197406 (Li et al. 2020); KIC 8197761 (Li et al. 2019a); KIC 9592855 (Guo, Gies \& Matson 2017); KIC 10080943 in which both components pulsate (Keen et al. 2015; Schmid \& Aerts 2016; Schmid et al. 2015); and KIC 10486425 (Zhang et al. 2018).

We also included the eclipsing binary KIC 2438249 which is located in the open cluster NGC 6791. We produced light curves for all 17 available quarters of KIC 2438249 using image subtraction photometry (Colman et al. in preparation), which involves the fine resampling and centroid realignment of every frame of image data, followed by the subtraction of an average to remove non-variable background flux.

In total, we found 35 binaries with clear $\gamma$ Dor-type patterns, comprising 15 stars from previous literature and 20 new stars. We list our results in Table 1. For the binary systems, we list the orbital periods, eccentricities, and pseudo-synchronous periods if eccentric. For the seismic analysis, we list the mode identifications $(l, k, m)$, asymptotic spacings, near-core rotation frequencies, the ranges of the radial orders, and the spin parameters. We also provide the ratio between the near-core and orbital periods, as well as the references for the stars that have been investigated. The amplitude spectra and period spacing patterns for all 35 binaries are shown in Appendix A. Since two components in KIC 10080943 and 12470041 pulsate, there are 37 figures in the appendix.

\subsection{Near-core rotation rates and asymptotic spacings}

We show the distribution of the near-core rotation rates of the 35 binaries in grey in Fig. 3. As a comparison, the near-core rotation rates of $611 \gamma$ Dor stars by $\mathrm{Li}$ et al. (2020) are also plotted as the yellow histogram.

The near-core rotation rates of the eclipsing binary sample are significantly lower than those from the 611-star sample by Li et al. (2020). For the latter, Li et al. (2020) reported that most stars rotate

${ }^{1}$ http://keplerebs.villanova.edu around $1 \mathrm{~d}^{-1}$, while some stars rotate slowly, forming an excess at the left end of the distribution. Conversely, the near-core rotations of the eclipsing binaries reported in this work are significantly slower, where the number decreases with increasing rotation rate. In a binary system where stars initially rotate faster than the orbital frequency, the tidal force decelerates the surface of the components and gradually slows down the near-core regions. In the 611-star sample by $\mathrm{Li}$ et al. (2020), there are still some binaries, such as the extremely shallow eclipsing binaries KIC 3341457 and KIC 8197761, the double-pulsator binary KIC 10080943, the PM binaries by Murphy et al. (2018), and the spectroscopic binary KIC 4480321 by Lampens et al. (2018). All the known $\gamma$ Dor stars with short orbital periods $\left(P_{\text {orb }}<20 \mathrm{~d}\right)$ show the near-core rotation rates slower than the peak value of the distribution from $\mathrm{Li}$ et al. (2020) in Fig. 3. Therefore, we speculate that the slow-rotator excess found by $\mathrm{Li}$ et al. (2020) might arise from non-eclipsing binaries with small semimajor axes.

The most rapid rotator in the eclipsing binary sample is KIC 4947528, whose near-core rotation rate is $2.017 \pm 0.020 \mathrm{~d}^{-1}$. The orbital period of KIC 4947528 is almost equal to the near-core rotation period, showing that the system has been tidally locked.

We also find three extremely slowly rotating stars. For these stars, the measurements of the near-core rotation rates have considerable uncertainties, because the slopes are typically small and are dominated by the fluctuations caused by the chemical abundance gradients, rather than the rotational effect (e.g. Miglio et al. 2008). However, we still confirm that these stars are rotating at extremely low rates. The slowest one is KIC 4142768, whose period spacing patterns have an average gradient of zero but show fluctuations and were reported by Guo et al. (2019). The near-core rotation rate of KIC 4142768 is $0.00037 \pm 0.00012 \mathrm{~d}^{-1}$, which is much lower than the orbital frequency $\left(\sim 0.071 \mathrm{~d}^{-1}\right)$. Aerts (2019) derived a near-core rotation rate $0.0060 \pm 0.0026 \mathrm{~d}^{-1}$ for KIC 4142768, which is faster than our value but is still very slow. KIC 9850387 is another extremely slow rotator, whose amplitude spectrum and period spacing patterns are shown in Fig. 4. The period spacings are almost identical, implying an extremely slow rotation rate, and we obtained a near-core rotation rate of $0.0053 \pm 0.0015 \mathrm{~d}^{-1}$ (see a recent study in Zhang et al. 2020). The last one is KIC 8197761, whose near-core rotation rate is $0.00336 \pm 0.00013 \mathrm{~d}^{-1}$, which was measured by rotational splittings by Sowicka et al. (2017) and Li et al. (2019a), hence shows a smaller uncertainty. Sowicka et al. (2017) made a spectroscopic observation of KIC 8197761 and found that the stellar surface is synchronized with the orbit, but the stellar core rotates $\sim 30$ times slower. The strong differential rotation is unexpected (e.g. Jermyn, Tayar \& Fuller 2020), and we will give a discussion in Section 3.6.

Fig. 5 displays the distributions of the asymptotic spacings, both for the eclipsing binary sample (grey) and the 611-star sample by Li et al. (2020, yellow). The distributions are similar, with the mean values both being around $4000 \mathrm{~s}$. KIC 11820830 has the smallest asymptotic spacing (2390 s), and its period spacing pattern shows a significant fluctuation, hence we infer that the star has evolved to the end of the main sequence. KIC 5809827 has the largest asymptotic spacing in our sample ( $9727 \mathrm{~s}$ ) and the star has nearly equal periods between the near-core region and the orbital motion. This star might be a very young star, since the asymptotic spacing decreases with stellar evolution. We considered whether it might be a Slowly Pulsating B (SPB) star since it shows a higher asymptotic spacing than $\gamma$ Dor stars (e.g. Pápics et al. 2017). However, the effective temperature given by Mathur et al. (2017) is $6369 \pm 226 \mathrm{~K}$, implying that it is not likely to be a B-type star. 


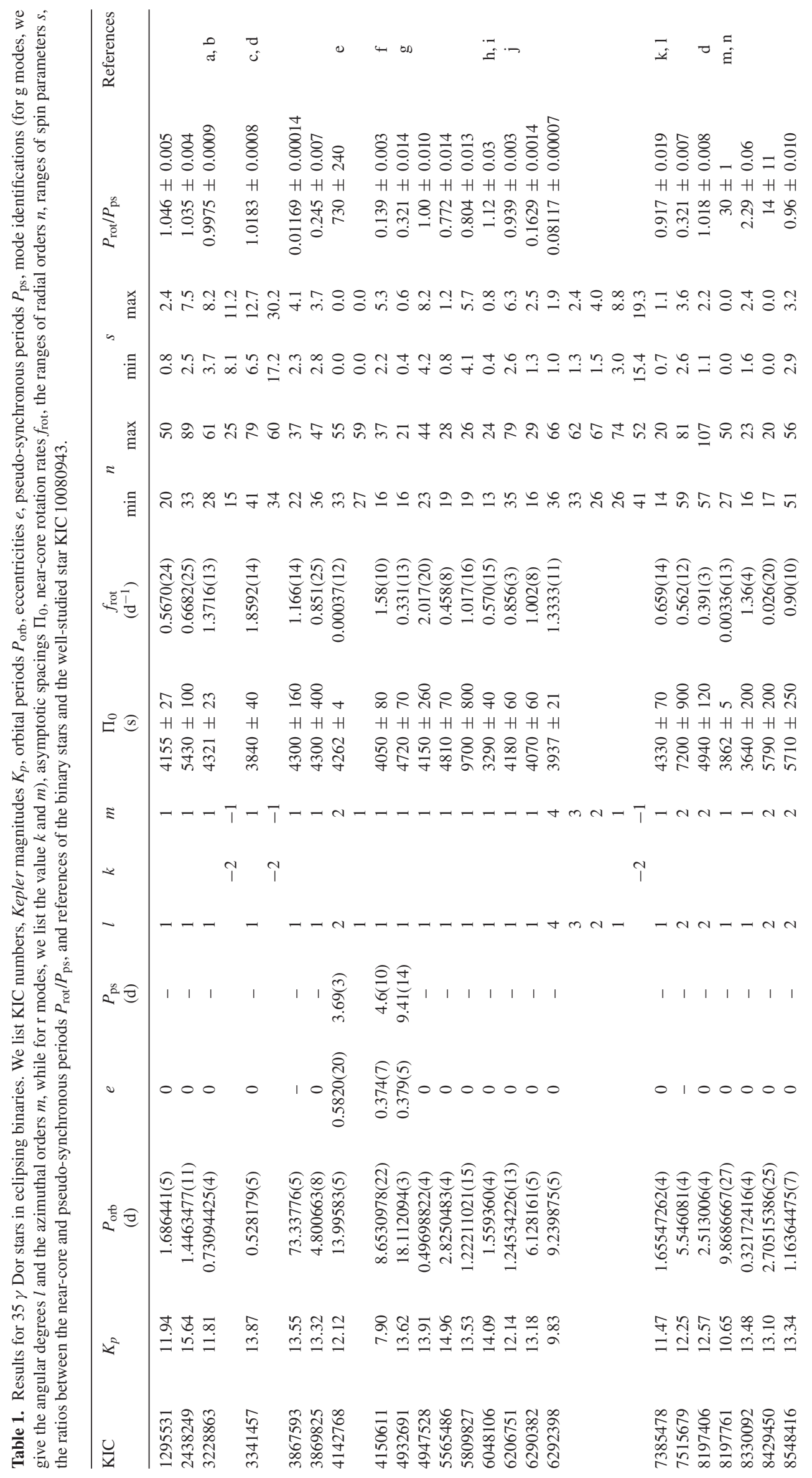




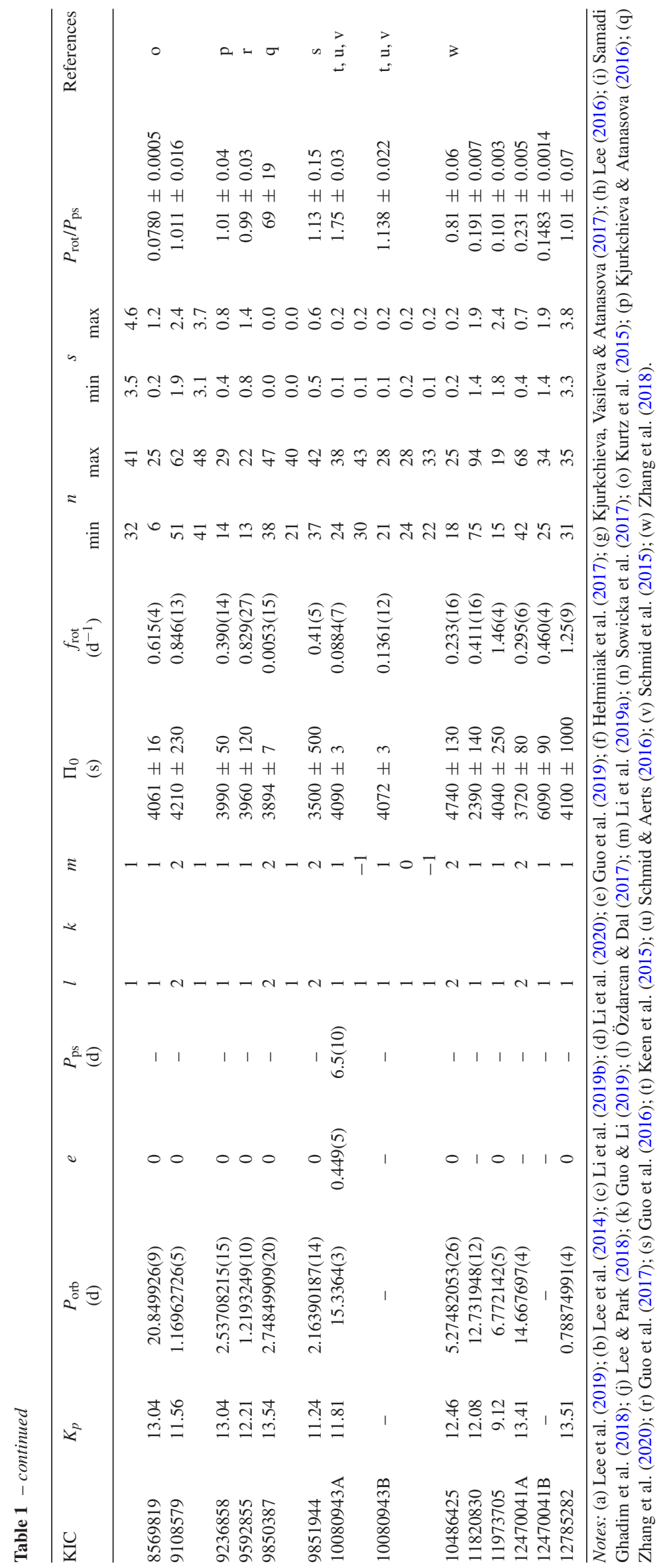




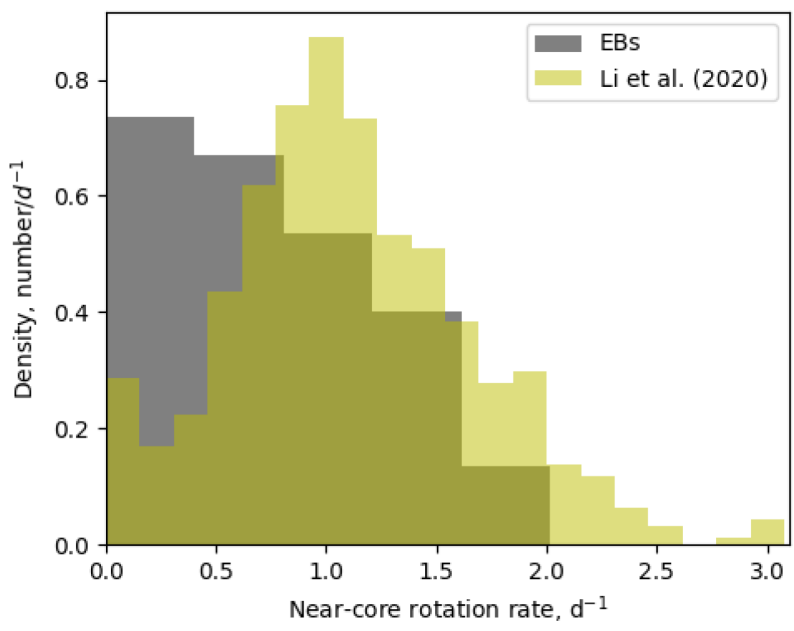

Figure 3. The distributions of the near-core rotation rates. The $y$-axis is normalized density so that the area under each histogram will sum to 1 . The grey histogram shows the distribution of the 35 binaries studied in this work. The yellow histogram contains $611 \gamma$ Dor stars by Li et al. (2020).

\subsection{The relation between the near-core and orbital periods}

Fig. 6 shows the relation between the near-core rotation periods and the orbital periods. For eccentric systems, we use the pseudo-synchronous periods $P_{\mathrm{ps}}$ (equation 1). We plot the 35 binaries (circles) by this work, and 45 phase-modulation (PM) binaries (squares) reported by Murphy et al. (2018) whose near-core rotations were measured using the g-mode patterns by Li et al. (2020). Due to the detection limit, the PM binaries typically have longer orbital periods from tens to thousands of days. The $\gamma$ Dor components of those systems are rotating freely since the tidal forces are weak, and they have near-core rotation rates around $1 \mathrm{~d}^{-1}$.

We classified the eclipsing binaries into three groups based on the ratio between the near-core and orbital periods $P_{\text {core }} / P_{\text {orb }}$.

(i) $P_{\text {core }} / P_{\text {orb }}<1$ : with the orbital periods around $10 \mathrm{~d}$, we see some eclipsing binaries whose near-core rotation periods are smaller than the orbital periods (below the red dotted line). These stars lie at the transition between the freely rotating and the tidally locked binaries, hence they have slightly higher near-core rotation periods than the PM binaries around $10^{3} \mathrm{~d}$, but not significantly.

(ii) $P_{\text {core }} / P_{\text {orb }} \approx 1$ : many stars in eclipsing binaries have almost the same orbital and near-core rotation periods, falling along the 1:1 straight line in the diagram (the red dotted line). We identify a tidally locked star if the rate of its near-core and orbital periods is between 0.7 and 1.3, which is indicated by the grey dashed box in Fig. 6 . Fig. 7 displays the period ratios of the tidally locked stars. We see that there is a smaller spread at the short-period side, presumably because the tidal force is stronger. The tidally locked star with the shortest orbital period is KIC 4947528. Binary offers an independent test of the TAR, and it proves that the TAR works effectively for dipole sectoral g modes with near-core rotation period of $0.5 \mathrm{~d}$, or with spin parameter up to 8 .

(iii) $P_{\text {core }} / P_{\text {orb }}>1$ : apart from three extremely slowly rotating stars introduced in Section 3.2, there are three other stars that have the near-core rotation periods substantially longer than their orbital periods. One is the well-studied star KIC 10080943, which is a non-eclipsing spectroscopic binary in which both components are $\gamma$ Dor pulsators (e.g. Keen et al. 2015; Schmid et al. 2015; Schmid \& Aerts 2016). For KIC 10080943B, the ratio between the near-core and pseudo-synchronous period is 1.14 , hence it is tidally locked. However, the ratio is 1.75 for KIC 10080943A, so this component is not pseudo-synchronous. The second one is KIC 8429450. This star only shows four g-mode peaks, hence there is a large uncertainty on the near-core rotation rate. However, it is not equal to the orbital period within the uncertainty range. The third star is KIC 8330092. This star has the shortest orbital period in the sample but its $\gamma$ Dor component is not synchronous. We will give a detailed discussion in Section 3.4. We also find that KIC 4480321 is a spectroscopic binary (Lampens et al. 2018), whose orbital period is $9.16592 \pm 0.00006 \mathrm{~d}$ and the near-core rotation rate is $121 \pm 4 \mathrm{~d}(\mathrm{Li}$ et al. 2019a). A hypothesis explaining the slow rotators is given in Section 3.6.

\subsection{KIC 8330092 with a pulsating third component}

As mentioned in Section 3.3, the binaries with orbital periods shorter than $10 \mathrm{~d}$ are likely to rotate synchronously in their nearcore regions. However, KIC 8330092 is an outlier. We checked the pixel files and did not find any contamination. We noticed that KIC 8330092 shows eclipse timing variations, hence there is a third companion (first reported by Conroy et al. 2014). To determine which component is pulsating, we applied the phase-modulation method to four p-mode peaks of this star (e.g. Murphy \& Shibahashi 2015; Murphy, Shibahashi \& Bedding 2016b; Hey et al. 2020). Fig. 8 displays the time delays of the eclipses and of the p-mode phases of KIC 8330092. The variations of $\mathrm{p}$-mode phases are the average of two p modes (19.674 and $\left.18.676 \mathrm{~d}^{-1}\right)$, measured with the PYTHON package MAELSTROM using 20-d subdivisions of the light curve. The primary eclipse timing variations were also binned every $20 \mathrm{~d}$. We find that the time delays of the eclipses and pulsations are anticorrelated, which means that the $\mathrm{p}$ modes are from the third component, rather than the close binary.

Using the Markov chain Monte Carlo (MCMC) time-delay modelling code in Murphy et al. (2016b), we obtain the orbital solutions of KIC 8330092 and its third component, as shown in Fig. 9. We find that the orbital period of the third component is $625 \pm 3 \mathrm{~d}$ with eccentricity $0.34 \pm 0.03$, and the combined mass of the binary system is $0.68 \pm 0.04$ times the mass of the third component. Considering that the third component is a $\delta$ Scuti star, the masses of the components in the binary are too low to be in the range of $\gamma$ Dor stars. Hence, we conclude that the g modes are also from the third component. The tidal force exerted on the third component is weak because the period of its orbit around the inner pair is long, so it is not surprising that synchronized rotation is not observed.

\subsection{KIC 12470041: both components have clear patterns}

We detected two period spacing patterns in the spectrum of KIC 12470041, as shown in Fig. 10. We cannot fit these two patterns simultaneously under the assumption that they come from the same component. Hence, KIC 12470041 is a system having two components that show period spacing patterns. In Fig. 10, the pattern around $0.7 \mathrm{~d}$ (dark grey area on the left) is identified as the $l=2, m$ $=2 \mathrm{~g}$ modes, since it shows a smaller slope compared to dipole $\mathrm{g}$ modes. We name this component KIC 12470041A. The peaks in the range from 1.1 to $1.6 \mathrm{~d}$ (dark grey area on the right) might be the $l$ $=1 \mathrm{~g}$ modes of KIC $12470041 \mathrm{~A}$, since they have a mean pulsation period twice the $l=2 \mathrm{~g}$ modes, as demonstrated by Saio et al. (2018b). However, we cannot identify any period spacings in them. The period spacings around $1 \mathrm{~d}$ (light grey area in the middle) are $l$ $=1, m=1 \mathrm{~g}$ modes of KIC 12470041B. 

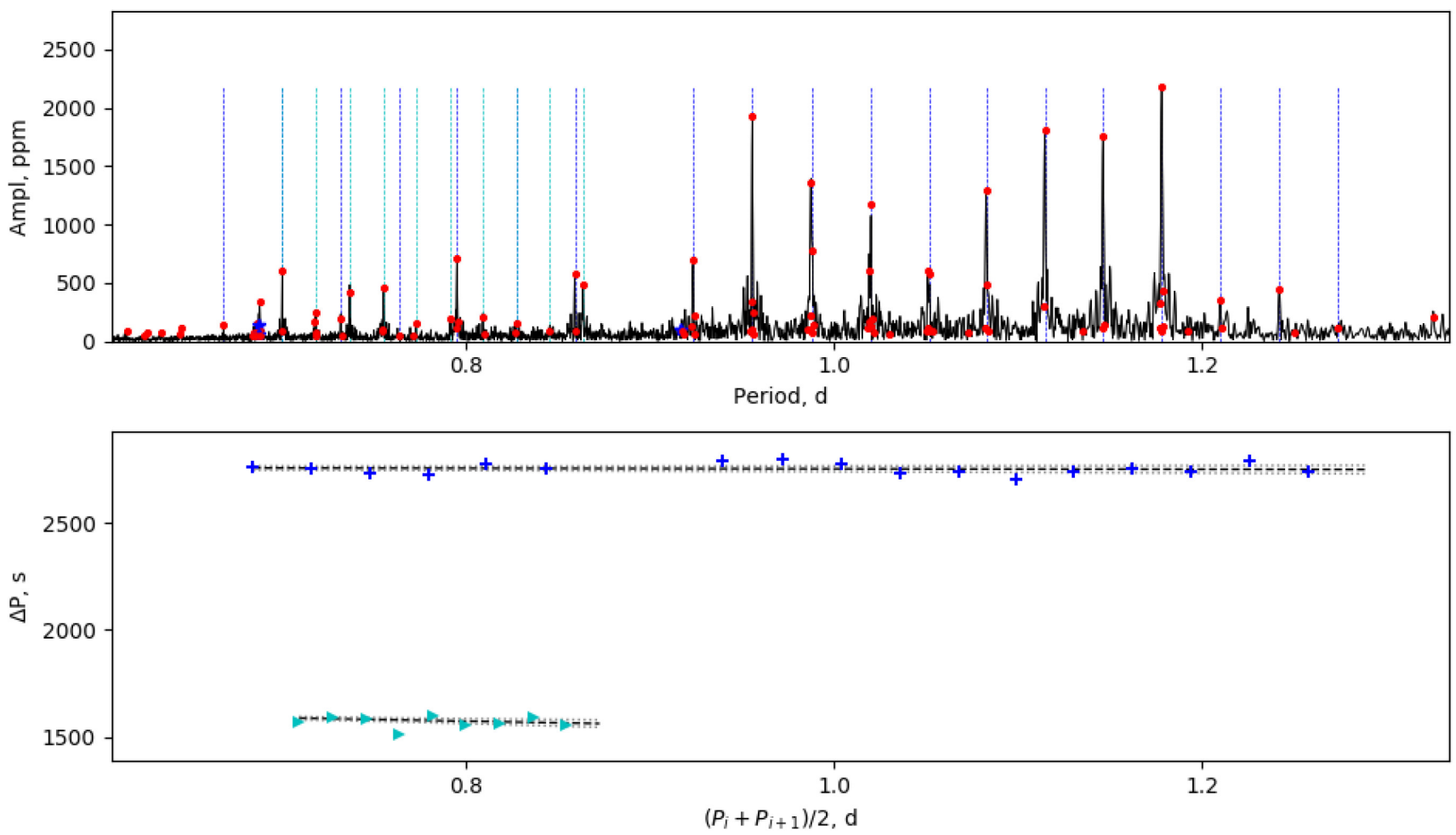

Figure 4. Top: the amplitude spectrum of KIC 9850387 after removing the eclipses from the light curve. The blue vertical dashed lines show the peaks of $l$ $=1 \mathrm{~g}$ modes, and the green vertical dashed lines mark the $l=2 \mathrm{~g}$ modes. The blue stars show the locations of orbital period harmonics. Bottom: the period spacings of $l=1$ and $2 \mathrm{~g}$ modes. The blue crosses are the pattern of $l=1 \mathrm{~g}$ modes and the green symbols are those of $l=2 \mathrm{~g}$ modes. The dashed lines show the best-fitting linear relation, whose uncertainties are shown by the grey dotted lines surrounding them.

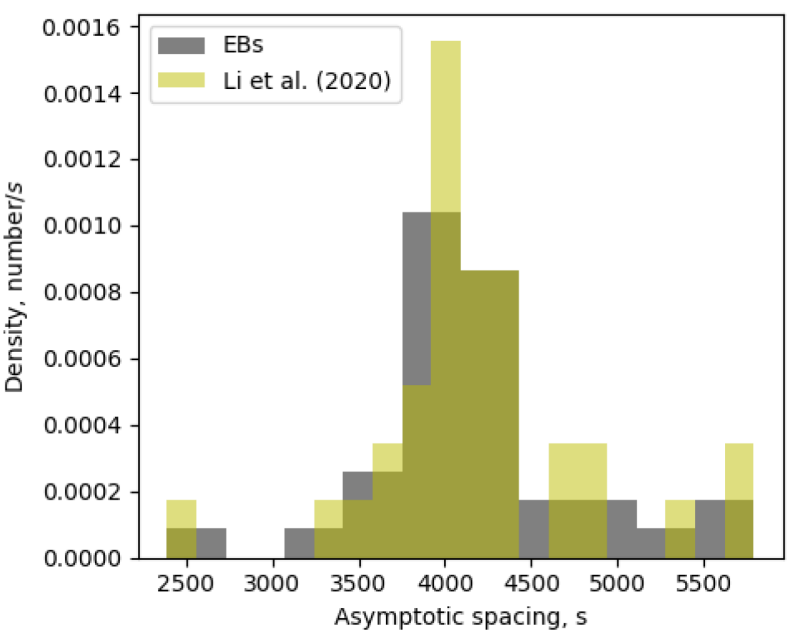

Figure 5. The distributions of the asymptotic spacings. The colours have the same meanings as Fig. 3.

We find that the asymptotic spacings of these two components differ greatly. The values are $3700 \mathrm{~s}$ for component A and $6090 \mathrm{~s}$ for component B. The asymptotic spacing decreases with stellar evolution, but stars with higher masses typically show higher asymptotic spacings. Since these two effects work in opposite directions, we cannot infer the evolutionary stages or masses of these two components straightforwardly in KIC 12470041 . The rotation rates of these two components are also different, and neither of them is tidally locked. Forward modelling in dynamics and asteroseismology would be needed to reveal the evolutionary stages.

\subsection{Inverse tide hypothesis}

In close binaries, stellar oscillations can be tidally excited. Such modes are typically high-order stellar g modes of frequency $\sim 1 \mathrm{~d}^{-1}$, in the same frequency range as the unstable pulsation modes that are linearly driven in the $\gamma$ Dor instability. In the literature, it is usually assumed that the tidally excited modes are damped modes that drive the system towards the lowest energy equilibrium state, namely that of a circular orbit with the spins of each star synchronized and aligned with the orbit. However, if tidally excited modes are unstable modes, the system may not evolve toward this minimum energy state. As we show below, the tidally excited modes may instead pump the eccentricity of the orbit or drive the stellar spins away from synchronism. We refer to this process as 'inverse tides.'

Following Fuller (2017, see also Burkart, Quataert \& Arras 2014), the angular momentum transferred from a tidally excited oscillation mode to its star is

$\dot{J}_{\alpha}=4 m \omega_{\alpha} \gamma_{\alpha}\left|a_{\alpha}\right|^{2} M R^{2}$,

where $m$ is the mode's azimuthal number, $\omega_{\alpha}$ is the mode frequency in the star's rotating frame, and $\gamma_{\alpha}$ is the mode damping rate. The mode amplitude is given by

$a_{\alpha}=\epsilon Q_{\alpha} F_{N m} \frac{\omega_{\alpha}}{\sqrt{\left(\omega_{\alpha}^{2}-\omega_{N m}\right)^{2}+\gamma_{\alpha}^{2}}}$.

Here, $\epsilon$ is the tidal forcing strength, $Q_{\alpha}$ is the mode quadrupole moment, and $F_{N m}$ is the temporal overlap of the forcing at an integer multiple $N$ of the orbital frequency. Definitions and conventions are described in Fuller (2017). In these expressions, $\omega_{\alpha}$ is defined to be positive (i.e. the negative-frequency modes have already been accounted for). We may consider the simplest case of a circular (but 


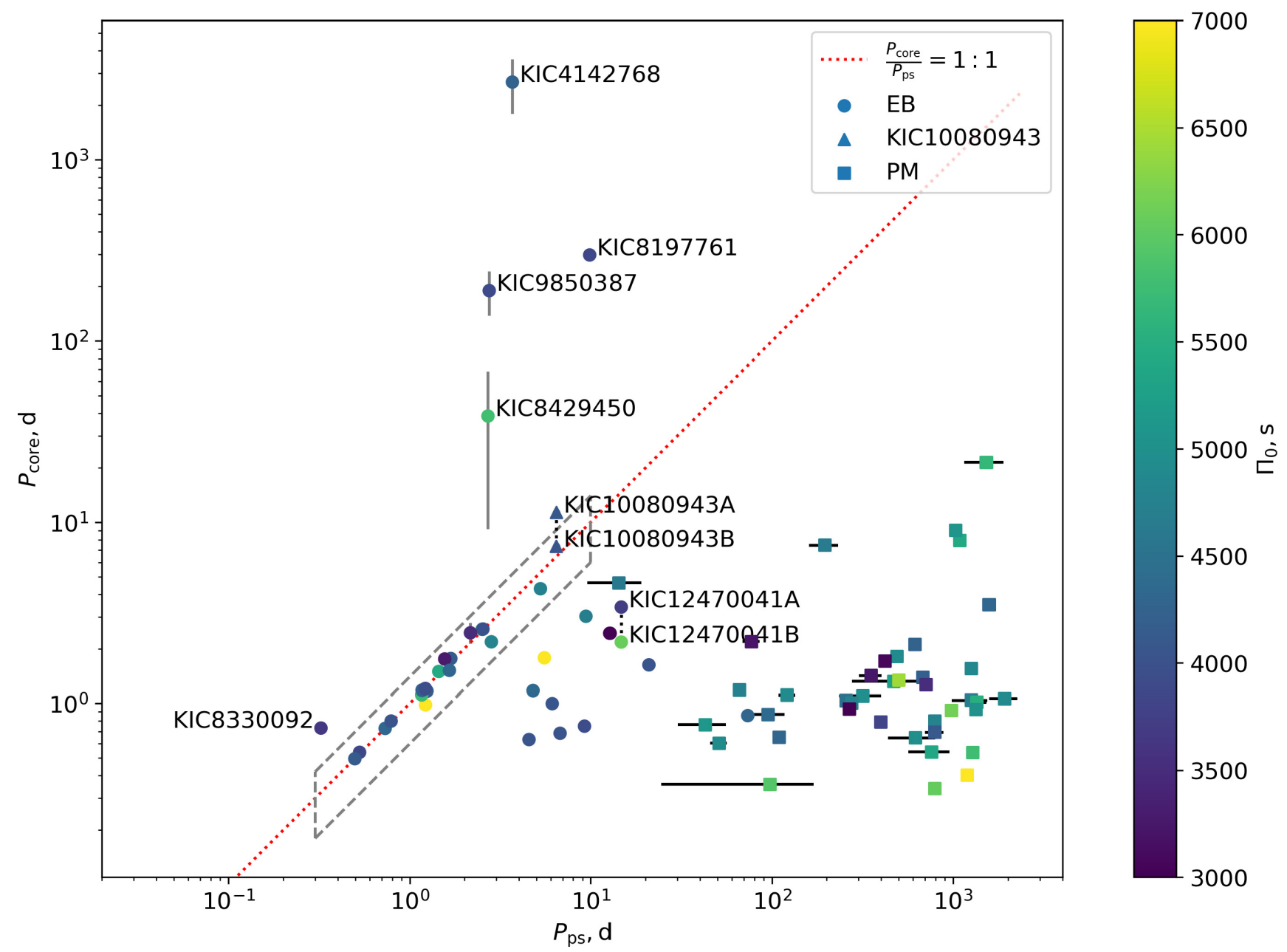

Figure 6. The relation between the near-core rotation periods $P_{\text {core }}$ and pseudo-synchronous periods $P_{\mathrm{ps}}$, coloured by their asymptotic spacings. For those whose eccentricities are zero or unknown, we use the orbital period instead of $P_{\mathrm{ps}}$. The circles are the eclipsing binaries reported by this work, while the squares are the PM binaries reported by Murphy et al. (2018). The dotted red line displays the location where the near-core rotation period is equal to the pseudo-synchronous period. The grey dashed lines enclose the tidally locked regions, whose $P_{\text {core }} / P_{\mathrm{ps}}$ is between 0.7 and 1.3 . Note that the boundaries of the colour bar are set between $3000 \mathrm{~s}$ and $7000 \mathrm{~s}$, hence the stars marked by light yellow might have asymptotic spacings larger than $7000 \mathrm{~s}$.

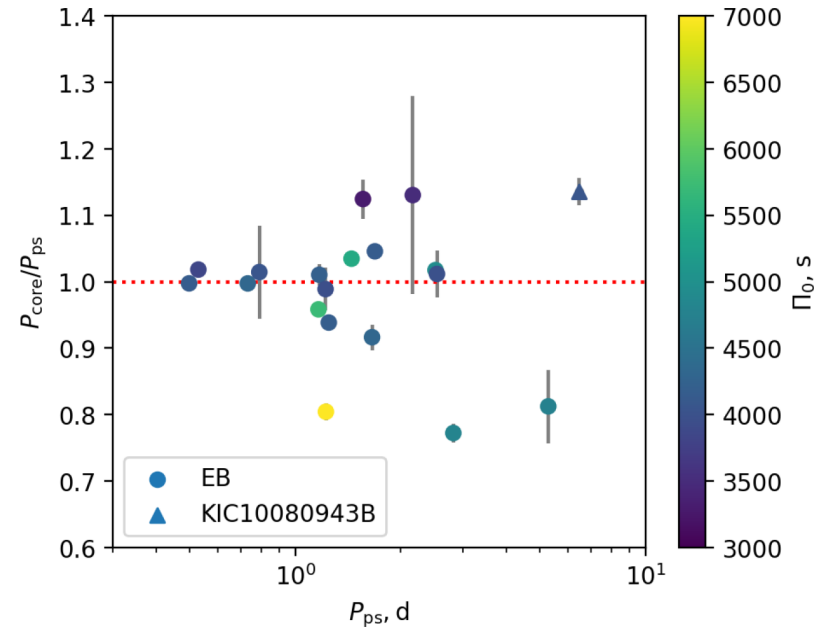

Figure 7. The ratios between the near-core and pseudo-synchronous periods of the tidally locked binaries shown in the grey box in Fig. 6, coloured by their asymptotic spacings. The boundaries of the colour bar are set between 3000 and $7000 \mathrm{~s}$.

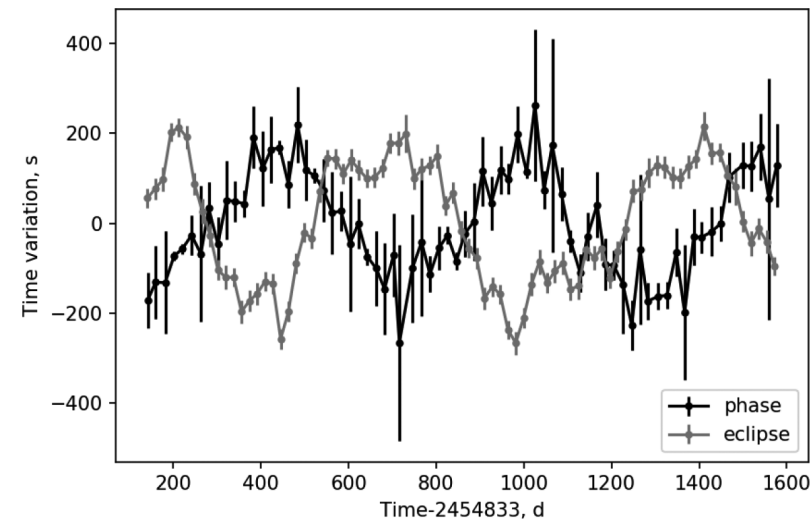

Figure 8. Time delays of the eclipses and the p-mode phases of KIC 8330092 .

not synchronized) orbit, for which tidal forcing only occurs at $N=m$ $=2$ in the case that the star rotates slower than the orbit (i.e. prograde modes are excited), or $-N=m=-2$ in the case that the star rotates faster than the orbit (i.e. retrograde modes are excited). 


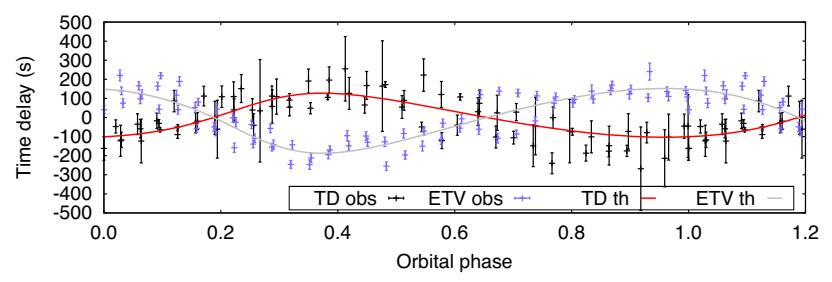

Figure 9. Phase-folded time delays of KIC 8330092. 'ETV' means the eclipsing time variation, and 'TD' means the time delay of the pulsating third component.

Considering equation (7), we see that the sign of angular momentum deposition in the star depends solely on the sign of $m \gamma_{\alpha}$, since all other terms are positive. In the case of a slowly rotating star with damped tidally excited oscillation modes (i.e. $m=2$ and $\gamma_{\alpha}>0$ ), the torque is positive and the star is spun up towards synchronism. For a rapidly rotating star with damped modes (i.e. $m=-2$ and $\gamma_{\alpha}$ $>0$ ), the torque on the star is negative and it is spun down towards synchronism.

However, consider now the case that the tidally excited modes are linearly unstable such that the damping rate $\gamma_{\alpha}$ is negative, as could easily be the case for $\gamma$ Dor stars in close binaries. In this case, the direction of angular momentum transfer will be opposite to the usual case: a slowly rotating star would be further spun down away from synchronism, and a rapidly rotating star would be further spun up away from synchronism. This inverse tidal effect could therefore cause binary $\gamma$ Dor stars to spin much slower or much faster than the orbital frequency. In both cases, the eccentricity of the orbit can be increased, and the system will move to a higher energy state, at the expense of the star's thermal energy that is pumped into the oscillation modes, and then transferred to the orbit.

Whether this inverse tidal mechanism can operate will depend on the combined contributions of all the tidally excited oscillations from both stars. A typical star will pulsate in both stable and unstable oscillation modes, and inverse tides can only operate if the unstable modes dominate the angular momentum transfer rate. This could certainly be achieved if the unstable modes are resonantly excited, as can occur in this sample of $\gamma$ Dor binaries, where orbital frequencies and the range of unstable mode frequencies overlap. The dynamics could be somewhat complex, including resonance locking processes (Witte \& Savonije 1999; Fuller et al. 2017). There could also exist systems where inverse tides operate in one star (acting to pump the orbit eccentricity and spin that star up or down), but normal tides operate in the other star (acting to damp the eccentricity and synchronize that star). We hope to explore such possibilities in future work.

Another important facet of the inverse tide hypothesis is the end state of the system. In our sample, we do not observe any close binaries that rotate much faster than the orbit (with the exception of the wide binaries, for which tidal processes are likely to be negligible). However, we do find a few stars whose cores rotate much slower than the orbit (there is another SPB star reported by Kallinger et al. 2017), perhaps indicating the inverse tide spin-down channel is more likely to occur in real systems. However, it is still not clear why the stars would be spun down to low rotation rates, since the inverse tidal process could easily spin them down to negative rotation rates, that is cause the stars to spin rapidly but retrograde relative to the orbit. In order for inverse tides to explain these observations,
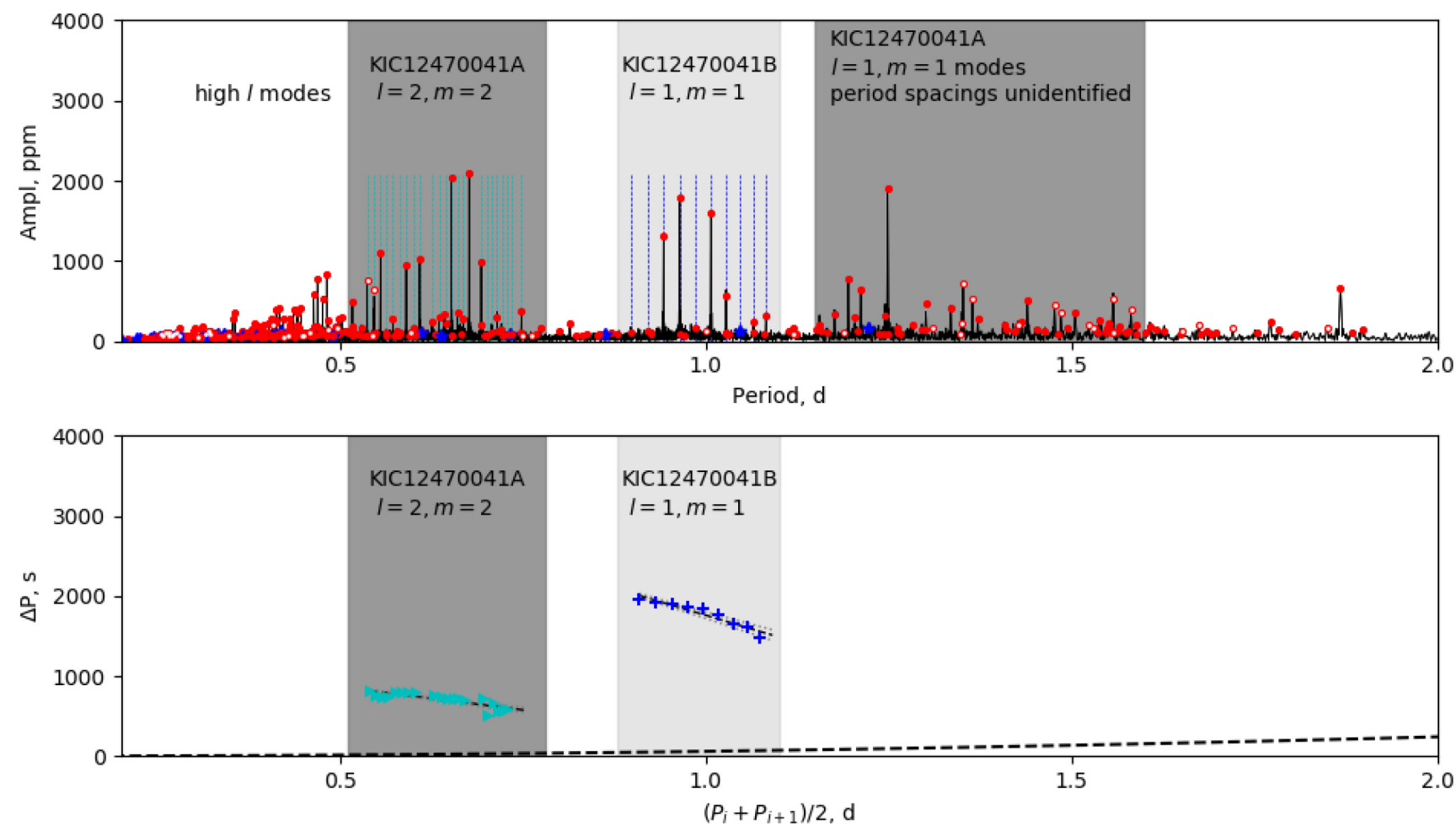

Figure 10. Amplitude spectrum and period spacing patterns of KIC 12470041. Top: amplitude spectrum. The dark grey areas mark the spectrum of KIC2470041A and the light grey area shows the spectrum of KIC 12470041B. The red points are the extracted frequencies, and the open dots are the harmonics, the blue stars mark the harmonics of the orbital period. Bottom: period spacing patterns. Note that the $l=1 \mathrm{~g}$-mode pattern of KIC $12470041 \mathrm{~A}$ is not identified. The dashed line shows the period resolution. 
there must be some mechanism which shuts off the process when the stellar spin frequency approaches zero. Interestingly, the observed stars with very slow rotation cluster at orbital periods around $5 \mathrm{~d}$, at the boundary between the tidally synchronized stars at shorter periods, and the longer period stars for which tides are negligible. This may indicate that inverse tides can only dominate over normal tides for these systems at intermediate orbital periods. There is much work to be done to investigate the possibility of inverse tides, but the very slowly rotating stars in this sample appear to indicate that tides can effectively spin-down the cores of some $\gamma$ Dor stars.

\subsection{Where are the dipole modes?}

Five stars show only quadrupole g-mode patterns in their amplitude spectra. Of these five, four stars (KIC 7515679, KIC 8197406, KIC 9851944, and KIC 12470041B) still show power excesses near the expected $l=1$ periods but the spectra are too crowded to identify any pattern. One star (KIC 10486425) does not have any power excess near the expected $l=1$ regions.

The occurrence rate is $5 / 35=14$ per cent. As a comparison, there are only 17 stars with missing dipole modes among the 611-star sample (2.8 per cent) reported by Li et al. (2020). The tidal effects of these five systems should be strong, since the longest orbital period is $\sim 14.67 \mathrm{~d}$ for KIC $12470041 \mathrm{~A}$ and the rest are below $\sim 6 \mathrm{~d}$. We infer that tidal effects are somehow encouraging quadrupole pulsations, but inhibiting dipole $g$ modes. Further theoretical explanation and observations are needed.

\section{CONCLUSIONS}

Tides affect the evolution and pulsation of close binaries. We searched for $\gamma$ Dor pulsators in eclipsing binaries with Kepler and found 35 binary systems that have g- or r-mode patterns. These stars provide the opportunity to reveal how tidal synchronization acts on the stellar interior in a binary system since we can measure the nearcore rotation rates and asymptotic spacings by their seismic signals.

The distribution of the near-core rotation rates of binaries is different from that of single stars. We find that the binaries tend to rotate more slowly, implying a significant tidal-locking effect on the $\gamma$ Dor components. We find that the near-core regions are more likely to be tidally locked if the orbital periods are shorter than $10 \mathrm{~d}$, and there are many stars with orbital periods around $10 \mathrm{~d}$ that lie at the transition between free rotation and synchronous rotation. Assuming tidal synchronization has occurred, the orbital period of the binary offers an independent test of the TAR, and we find that the near-core rotation periods given by the TAR are in agreement with the orbital periods down to rotation periods of $0.5 \mathrm{~d}$, or spin parameters up to 8 .

We also find that three stars are rotating extremely slowly, which may signal the operation of a new mechanism we refer to as 'inverse tides'. The classical tides excite damped oscillation modes, which lead to synchronization and circularization. However, in the 'inverse tides' situation, the tidally-excited modes are unstable, allowing for angular momentum transfer that drives the system away from synchronism rather than toward it. There are still many questions that remain, such as when 'inverse tides' can operate and the end state of this process. It is indeed a new aspect of tides waiting for more investigation.

\section{ACKNOWLEDGEMENTS}

This work was supported by the Australian Research Council through DECRA DE180101104. Funding for the Stellar Astrophysics Centre is provided by the Danish National Research Foundation (grant agreement no.: DNRF106). This work was partially supported by funding from the Center for Exoplanets and Habitable Worlds. The Center for Exoplanets and Habitable Worlds is supported by the Pennsylvania State University, the Eberly College of Science, and the Pennsylvania Space Grant Consortium. We appreciate Andrej Prša and Diana Windemuth for providing eccentricities of some EBs. We appreciate Adam Jermyn for sharing his paper draft before publication.

\section{DATA AVAILABILITY}

The light curves were downloaded from the public data archive at MAST, ${ }^{2}$ using the PYTHON package LIGHTKURVE (Lightkurve Collaboration et al. 2018). ${ }^{3}$ The Kepler eclipsing binary catalogue (third revision) $)^{4}$ provided some basic parameters of some eclipsing binaries. The scientific output is available in Table 1 in the article and in its online supplementary material.

\section{REFERENCES}

Aerts C., 2019, preprint (arXiv:1912.12300)

Andersen J., 1991, A\&AR, 3, 91

Balona L. A., Krisciunas K., Cousins A. W. J., 1994, MNRAS, 270, 905

Berthomieu G., Gonczi G., Graff P., Provost J., Rocca A., 1978, A\&A, 70, 597

Borucki W. J. et al., 2010, Science, 327, 977

Bouabid M.-P., Dupret M.-A., Salmon S., Montalbán J., Miglio A., Noels A., 2013, MNRAS, 429, 2500

Breger M. et al., 1993, A\&A, 271, 482

Burkart J., Quataert E., Arras P., 2014, MNRAS, 443, 2957

Conroy K. E., Prša A., Stassun K. G., Orosz J. A., Fabrycky D. C., Welsh W. F., 2014, AJ, 147, 45

Czesla S., Terzenbach S., Wichmann R., Schmitt J. H. M. M., 2019, A\&A, 623, A107

del Burgo C., Allende Prieto C., 2018, MNRAS, 479, 1953

Doyle L. R. et al., 2011, Science, 333, 1602

Duchêne G., Kraus A., 2013, ARA\&A, 51, 269

Eckart C., 1960, Hydrodynamics of Oceans and Atmospheres. Pergamon Press, Oxford, http://cds.cern.ch/record/1975181

Faulkner J., 1971, ApJ, 170, L99

Fuller J., 2017, MNRAS, 472, 1538

Fuller J., Hambleton K., Shporer A., Isaacson H., Thompson S., 2017, MNRAS, 472, L25

Gaulme P., Guzik J. A., 2019, A\&A, 630, A106

Goldreich P., Nicholson P. D., 1989a, ApJ, 342, 1075

Goldreich P., Nicholson P. D., 1989b, ApJ, 342, 1079

Guo Z., Li G., 2019, ApJ, 882, L5

Guo Z., Gies D. R., Matson R. A., García Hernández A., 2016, ApJ, 826, 69

Guo Z., Gies D. R., Matson R. A., 2017, ApJ, 851, 39

Guo Z., Fuller J., Shporer A., Li G., Hambleton K., Manuel J., Murphy S., Isaacson H., 2019, ApJ, 885, 46

Guszejnov D., Hopkins P. F., Krumholz M. R., 2017, MNRAS, 468, 4093

Hambleton K. M. et al., 2013, MNRAS, 434, 925

Hełminiak K. G., Ukita N., Kambe E., Kozłowski S. K., Pawłaszek R., Maehara H., Baranec C., Konacki M., 2017, A\&A, 602, A30

Hey D. R., Murphy S. J., Foreman-Mackey D., Bedding T. R., Pope B. J. S., Hogg D. W., 2020, AJ, 159, 20

Hilditch R. W., Howarth I. D., Harries T. J., 2005, MNRAS, 357, 304

Hut P., 1981, A\&A, 99, 126

Hypolite D., Rieutord M., 2014, A\&A, 572, A15

\footnotetext{
${ }^{2}$ https://mast.stsci.edu/

${ }^{3}$ https://docs.lightkurve.org/index.html

${ }^{4}$ http://keplerebs.villanova.edu
} 
Jermyn A. S., Tayar J., Fuller J., 2020, MNRAS, 491, 690

Kallinger T. et al., 2017, A\&A, 603, A13

Kaye A. B., Handler G., Krisciunas K., Poretti E., Zerbi F. M., 1999, PASP, 111,840

Keen M. A., Bedding T. R., Murphy S. J., Schmid V. S., Aerts C., Tkachenko A., Ouazzani R.-M., Kurtz D. W., 2015, MNRAS, 454, 1792

Kirk B. et al., 2016, AJ, 151, 68

Kjurkchieva D., Atanasova T., 2016, New Astron., 49, 17

Kjurkchieva D., Vasileva D., Atanasova T., 2017, AJ, 154, 105

Koch D. G. et al., 2010, ApJ, 713, L79

Kurtz D. W., Saio H., Takata M., Shibahashi H., Murphy S. J., Sekii T., 2014, MNRAS, 444, 102

Kurtz D. W., Hambleton K. M., Shibahashi H., Murphy S. J., Prša A., 2015, MNRAS, 446, 1223

Lampens P. et al., 2018, A\&A, 610, A17

Lee U., 1993, ApJ, 417, 697

Lee J. W., 2016, ApJ, 833, 170

Lee J. W., Park J.-H., 2018, MNRAS, 480, 4693

Lee U., Saio H., 1997, ApJ, 491, 839

Lee J. W., Kim S.-L., Hong K., Lee C.-U., Koo J.-R., 2014, AJ, 148, 37

Lee J. W., Hong K., Koo J.-R., Park J.-H., 2019, AJ, 159, 24

Li G., Bedding T. R., Murphy S. J., Van Reeth T., Antoci V., Ouazzani R.-M., 2019a, MNRAS, 482, 1757

Li G., Van Reeth T., Bedding T. R., Murphy S. J., Antoci V., 2019b, MNRAS, 487,782

Li G., Van Reeth T., Bedding T. R., Murphy S. J., Antoci V., Ouazzani R.-M., Barbara N. H., 2020, MNRAS, 491, 3586

Lightkurve Collaboration et al., 2018, Astrophysics Source Code Library, record ascl:1812.013

Mathur S. et al., 2017, ApJS, 229, 30

Matijevič G., Prša A., Orosz J. A., Welsh W. F., Bloemen S., Barclay T., 2012, AJ, 143, 123

Matson R. A., Gies D. R., Guo Z., Orosz J. A., 2016, AJ, 151, 139

Maxted P. F. L., et al., 2020, MNRAS, in press

Miglio A., Montalbán J., Noels A., Eggenberger P., 2008, MNRAS, 386, 1487

Moe M., Di Stefano R., 2017, ApJS, 230, 15

Murphy S. J., 2018, preprint (arXiv:1811.12659)

Murphy S. J., Shibahashi H., 2015, MNRAS, 450, 4475

Murphy S. J., Fossati L., Bedding T. R., Saio H., Kurtz D. W., Grassitelli L., Wang E. S., 2016a, MNRAS, 459, 1201

Murphy S. J., Shibahashi H., Bedding T. R., 2016b, MNRAS, 461, 4215

Murphy S. J., Moe M., Kurtz D. W., Bedding T. R., Shibahashi H., Boffin H. M. J., 2018, MNRAS, 474, 4322

North P., Gauderon R., Barblan F., Royer F., 2010, A\&A, 520, A74

Ouazzani R.-M., Salmon S. J. A. J., Antoci V., Bedding T. R., Murphy S. J., Roxburgh I. W., 2017, MNRAS, 465, 2294

Papaloizou J., Pringle J. E., 1978, MNRAS, 182, 423

Pápics P. I. et al., 2017, A\&A, 598, A74

Provost J., Berthomieu G., Rocca A., 1981, A\&A, 94, 126

Prša A. et al., 2011, AJ, 141, 83

Rieutord M., 2006, A\&A, 451, 1025

Rossby C. G., 1939, J. Mar. Res., 2, 38

Saio H., 1982, ApJ, 256, 717

Saio H., 2019, in Neiner C., Weiss W. W., Baade D., Griffin R. E., Lovekin C. C., Moffat A. F. J., eds, Proceedings of the Conference Stars and their Variability Observed from Space, held in Vienna on August 19-23, 2019. University of Vienna, Vienna, Austria, p. 321

Saio H., Kurtz D. W., Takata M., Shibahashi H., Murphy S. J., Sekii T., Bedding T. R., 2015, MNRAS, 447, 3264

Saio H., Kurtz D. W., Murphy S. J., Antoci V. L., Lee U., 2018a, MNRAS, 474,2774

Saio H., Bedding T. R., Kurtz D. W., Murphy S. J., Antoci V., Shibahashi H., Li G., Takata M., 2018b, MNRAS, 477, 2183

Samadi Ghadim A., Lampens P., Jassur M., 2018, MNRAS, 474, 5549

Schmid V. S. et al., 2015, A\&A, 584, A35

Schmid V. S., Aerts C., 2016, A\&A, 592, A116

Shporer A. et al., 2016, ApJ, 829, 34
Slawson R. W. et al., 2011, AJ, 142, 160

Sowicka P., Handler G., Dębski B., Jones D., Van de Sand e M., Pápics P. I., 2017, MNRAS, 467, 4663

Sriram K., Malu S., Choi C. S., Vivekananda Rao P., 2017, AJ, 153, 231

Sterken C., 2005, in Sterken C., ed., ASP Conf. Ser. Vol. 335, The Light-Time Effect in Astrophysics: Causes and cures of the O-C diagram. Astron. Soc. Pac., Valley University, p. 3

Takata M., Ouazzani R. M., Saio H., Christophe S., Ballot J., Antoci V., Salmon S. J. A. J., Hijikawa K., 2020, A\&A, 635, A106

Torres G., Ribas I., 2002, ApJ, 567, 1140

Townsend R. H. D., 2003, MNRAS, 340, 1020

Townsend R. H. D., Teitler S. A., 2013, MNRAS, 435, 3406

Townsend R. H. D., Goldstein J., Zweibel E. G., 2018, MNRAS, 475, 879

Van Reeth T. et al., 2015, ApJS, 218, 27

Van Reeth T. et al., 2018, A\&A, 618, A24

Van Reeth T., Tkachenko A., Aerts C., 2016, A\&A, 593, A120

Welsh W. F. et al., 2011, ApJS, 197, 4

Witte M. G., Savonije G. J., 1999, A\&A, 350, 129

Zahn J. P., 1975, A\&A, 41, 329

Zahn J. P., 1977, A\&A, 500, 121

Zhang X. B., Fu J. N., Luo C. Q., Ren A. B., Yan Z. Z., 2018, ApJ, 865, 115

Zhang X., Chen X., Zhang H., Fu J., Li Y., 2020, ApJ, 895, 124

Özdarcan O., Dal H. A., 2017, PASA, 34, e017

\section{SUPPORTING INFORMATION}

Supplementary data are available at MNRAS online.

Figure A1. The period spacing patterns of KIC 4947528. Figure A2. The period spacing patterns of KIC 3341457.

Figure A3. The period spacing patterns of KIC 4150611.

Figure A4. The period spacing patterns of KIC 11973705.

Figure A5. The period spacing patterns of KIC 3228863.

Figure A6. The period spacing patterns of KIC 8330092.

Figure A7. The period spacing patterns of KIC 6292398.

Figure A8. The period spacing patterns of KIC 12785282.

Figure A9. The period spacing patterns of KIC 3867593.

Figure A10. The period spacing patterns of KIC 5809827.

Figure A11. The period spacing patterns of KIC 6290382.

Figure A12. The period spacing patterns of KIC 8548416.

Figure A13. The period spacing patterns of KIC 6206751.

Figure A14. The period spacing patterns of KIC 3869825.

Figure A15. The period spacing patterns of KIC 9108579.

Figure A16. The period spacing patterns of KIC 9592855.

Figure A17. The period spacing patterns of KIC 2438249.

Figure A18. The period spacing patterns of KIC 7385478.

Figure A19. The period spacing patterns of KIC 8569819.

Figure A20. The period spacing patterns of KIC 6048106.

Figure A21. The period spacing patterns of KIC 1295531.

Figure A22. The period spacing patterns of KIC 7515679.

Figure A23. The period spacing patterns of KIC 12470041B.

Figure A24. The period spacing patterns of KIC 5565486.

Figure A25. The period spacing patterns of KIC 11820830.

Figure A26. The period spacing patterns of KIC 9851944.

Figure A27. The period spacing patterns of KIC 8197406.

Figure A28. The period spacing patterns of KIC 9236858.

Figure A29. The period spacing patterns of KIC 4932691.

Figure A30. The period spacing patterns of KIC 12470041A.

Figure A31. The period spacing patterns of KIC 10486425.

Figure A32. The period spacing patterns of KIC 10080943B.

Figure A33. The period spacing patterns of KIC 10080943A.

Figure A34. The period spacing patterns of KIC 8429450.

Figure A35. The period spacing patterns of KIC 9850387. 
Figure A36. The period spacing patterns of KIC 8197761. Figure A37. The period spacing patterns of KIC 4142768.

Please note: Oxford University Press is not responsible for the content or functionality of any supporting materials supplied by the authors. Any queries (other than missing material) should be directed to the corresponding author for the article.

\section{APPENDIX A: AMPLITUDE SPECTRA AND PERIOD SPACING PATTERNS}

We display the amplitude spectra and period spacing patterns of the $\gamma$ Dor stars reported by this work, sorted by their descending near-core rotation rates. For each figure, the top panel shows the amplitude spectrum with $x$-axis of pulsation period. The extracted peaks are marked by red dots, and the likely orbital period harmonics are marked by blue stars. The identified g- or r-mode peaks are marked by the vertical lines. The bottom panel shows the period spacings, whose $x$-axis is the mean period. We also give the linear fits of the period spacings as a function of period, as shown by the dashed lines, whose uncertainty are given by the dotted lines surrounding it.

This paper has been typeset from a $\mathrm{T}_{\mathrm{E}} \mathrm{X} / \mathrm{L} \mathrm{T} \mathrm{E} \mathrm{X}$ file prepared by the author. 